DRAFT VERSION MARCH 10, 2020

Preprint typeset using IATEX $_{\mathrm{E}}$ style AASTeX6 v. 1.0

\title{
ON THE OPENING ANGLE OF MAGNETISED JETS FROM NEUTRON-STAR MERGERS: THE CASE OF GRB170817A
}

\author{
Antonios Nathanail ${ }^{1}$, Ramandeep Gill $^{2,3}$, Oliver Porth ${ }^{4}$, Christian M. Fromm ${ }^{1,5}$, Luciano Rezzolla ${ }^{1,6}$ \\ ${ }^{1}$ Institut für Theoretische Physik, Max-von-Laue-Strasse 1, 60438 Frankfurt, Germany \\ ${ }^{2}$ Department of Physics, The George Washington University, Washington, DC 20052, USA \\ ${ }^{3}$ Department of Natural Sciences, The Open University of Israel, 1 University Road, PO Box 808, Raanana 4353701, Israel \\ ${ }^{4}$ Astronomical Institute Anton Pannekoek, Universeit van Amsterdam, Science Park 904, 1098 XH, Amsterdam, The Netherlands \\ ${ }^{5}$ Max-Planck-Institut für Radioastronomie, Auf dem Hügel 69, D-53121 Bonn, Germany \\ ${ }^{6}$ School of Mathematics, Trinity College, Dublin 2, Ireland
}

\begin{abstract}
The observations of GW170817/GRB170817A have confirmed that the coalescence of a neutron-star binary is the progenitor of a short gamma-ray burst. In the standard picture of a short gammaray burst, a collimated highly relativistic outflow is launched after merger and it successfully breaks out from the surrounding ejected matter. Using initial conditions inspired from numerical-relativity binary neutron-star merger simulations, we have performed general-relativistic hydrodynamic (HD) and magnetohydrodynamic (MHD) simulations in which the jet is launched and propagates selfconsistently. The complete set of simulations suggests that: (i) MHD jets have an intrinsic energy and velocity polar structure with a "hollow core" subtending an angle $\theta_{\text {core }} \approx 4^{\circ}-5^{\circ}$ and an opening angle of $\theta_{\text {jet }}>10^{\circ}$; (ii) MHD jets eject significant amounts of matter and two orders of magnitude more than HD jets; (iii) the energy stratification in MHD jets naturally yields the power-law energy scaling $E(>\Gamma \beta) \propto(\Gamma \beta)^{-4.5}$; (iv) MHD jets provide fits to the afterglow data from GRB170817A that are comparatively better than those of the HD jets and without free parameters; $(v)$ finally, both of the best-fit $\mathrm{HD} / \mathrm{MHD}$ models suggest an observation angle $\theta_{\mathrm{obs}} \simeq 21^{\circ}$ for GRB170817A.
\end{abstract}

Keywords: Gamma-ray bursts, Neutron stars, Magnetohydrodynamics

\section{INTRODUCTION}

The first detection of gravitational waves (GWs) from a binary neutron-star (BNS) merger, GW170817 (The LIGO Scientific Collaboration \& The Virgo Collaboration 2017), was marked by a coincident detection of a short gamma-ray burst (GRB), GRB170817A (Savchenko et al. 2017; Goldstein et al. 2017). This was followed by observations across the electromagnetic (EM) spectrum, with the detection of the (The LIGO Scientific Collaboration et al. 2017) quasi-thermal kilonova emission in UV, optical, and NIR followed by the delayed detection of the non-thermal afterglow emission in the X- $(t>8.9 \mathrm{~d}$; Troja et al. 2017), optical, and radio $(t>16.4 \mathrm{~d}$; Hallinan et al. 2017) bands.

The continuous brightening of the broadband afterglow flux, with its peculiar shallow rise $\left(F_{v} \propto t^{0.8}\right)$ to the peak at $t_{\mathrm{pk}} \simeq 150 \mathrm{~d}$ post-merger (Lyman et al. 2018; Margutti et al. 2018; Mooley et al. 2018a), was interpreted using two main models. The first one considered a "structured outflow" (e.g, Gill \& Granot 2018), namely, a polar-structured jet with a narrow relativistic core sur- rounded by low-energy wings (e.g., Troja et al. 2017, 2018; D'Avanzo et al. 2018; Margutti et al. 2018; Lazzati et al. 2018). The second model considered a "cocoon", namely, a wide-angle outflow expanding quasi-spherically and with radial velocity stratification (e.g., Kasliwal et al. 2017; Gottlieb et al. 2018; Mooley et al. 2018a). The subsequent observation of apparent superluminal motion of the radio flux centroid (Mooley et al. 2018b), together with the compact size of the radio image (i.e., $\lesssim 2$ mas) (Ghirlanda et al. 2019), strongly favored the structured jet model as dominating the afterglow emission near and post $t_{\mathrm{pk}}$.

Numerical and semi-analytical models of hydrodynamic jets have been employed to explore the afterglow of GRB170817A and the models that best fit the afterglow data correspond to structured jets with angular size of the relativistic core of $\sim 3^{\circ}-5^{\circ}$ (Mooley et al. 2018b; Ghirlanda et al. 2019; Troja et al. 2019).

Most of the analysis for the outflow of GRB170817A has been done using semi-analytical models or relativistic hydrodynamic simulations that launch a jet far from the merger site, with launching radius of $10^{9} \mathrm{~cm}$. These 


\begin{tabular}{|c|c|c|c|c|c|c|c|c|c|c|c|c|c|c|}
\hline model & $\begin{array}{c}L \\
{[\mathrm{erg} / \mathrm{s}]}\end{array}$ & $\begin{array}{l}t_{\text {inj }} \\
{[\mathrm{s}]}\end{array}$ & $\Gamma_{\text {init }}$ & $\begin{array}{c}\theta_{\text {jet }} \\
{[\mathrm{deg}]}\end{array}$ & $\begin{array}{l}E_{B_{\phi}} \\
{[\mathrm{erg}]} \\
10^{49}\end{array}$ & $\begin{array}{l}E_{B_{\mathrm{p}}} \\
{[\mathrm{erg}]} \\
10^{49} \\
\end{array}$ & $\frac{E_{B_{\mathrm{p}}}}{E_{B_{\phi}}}$ & $\sigma_{\max }$ & $\beta_{\min }$ & $\begin{array}{c}\rho_{\max } \\
{\left[\mathrm{g} / \mathrm{cm}^{3}\right]} \\
10^{10}\end{array}$ & $a$ & $\begin{array}{c}M_{\mathrm{tot}} \\
{\left[M_{\odot}\right]}\end{array}$ & $\begin{array}{c}M_{\mathrm{ej}} \\
{\left[M_{\odot}\right]}\end{array}$ & $\begin{array}{c}\frac{M_{\mathrm{ej}}}{M_{\mathrm{tot}}} \\
\%\end{array}$ \\
\hline HD-tht. 6 & $10^{51}$ & 0.1 & 10 & 6 & - & - & - & - & - & 1.5 & 0.9375 & 0.108 & 0.0001 & 0.12 \\
\hline HD-tht. 3 & $10^{51}$ & 0.1 & 10 & 3 & - & - & - & - & - & 1.5 & 0.9375 & 0.108 & 0.0001 & 0.13 \\
\hline MHD-p2t.03 & - & - & - & - & 5.0 & 1.6 & 0.3 & 0.065 & 0.13 & 1.5 & 0.9375 & 0.108 & 0.039 & 36.0 \\
\hline MHD-p2t. 02 & - & - & - & - & 10 & 2.1 & 0.2 & 0.065 & 0.13 & 2.0 & 0.9375 & 0.144 & 0.053 & 37.1 \\
\hline MHD-p2t. 12 & - & - & - & - & 1.2 & 1.5 & 1.2 & 0.036 & 0.13 & 1.5 & 0.9375 & 0.108 & 0.036 & 34.1 \\
\hline
\end{tabular}

Table 1. Properties of the various HD and MHD jets considered: luminosity of the HD jet $(L)$, duration of the HD injection $\left(t_{\text {inj }}\right)$, initial Lorentz factor of the $\mathrm{HD}$ jet $\left(\Gamma_{\text {init }}\right)$, initial opening angle of the HD jet $\left(\theta_{\text {jet }}\right)$, toroidal and poloidal magnetic energies $\left(E_{B_{\phi}}, E_{B_{\mathrm{p}}}\right)$ and their ratio, maximum magnetization in the torus $\left(\sigma:=B^{2} / 4 \pi \rho\right)$, minimum plasma parameter in torus $\left(\beta:=p / p_{m}\right.$, where $p$ and $p_{m}$ are the fluid and magnetic pressures respectively), maximum density of the torus $\left(\rho_{\max }\right)$ and dimensionless spin parameter of the $\mathrm{BH}\left(a:=J / M^{2}\right)$, initial total mass $\left(M_{\mathrm{tot}}\right)$, ejected mass $\left(M_{\mathrm{ej}}\right)$ and their ratio.

hydrodynamic studies have been accompanied by much fewer investigations making use of MHD simulations to study the properties of such outflows (Kathirgamaraju et al. 2018; Bromberg et al. 2018; Geng et al. 2019), and in two cases, the jets were launched self-consistently via the accretion and rotation of the black hole (Fernández et al. 2018; Kathirgamaraju et al. 2019). In addition to such self-consistent evolutions, Kathirgamaraju et al. 2019 were also the first to report afterglow lightcurves as derived from the MHD simulations.

We here report on a series of two-dimensional (2D) general-relativistic MHD (GRMHD) simulations of jets that are self-consistently launched after a BNS merger when the merger remnant has collapsed to a black hole (BH). In addition, we also carry out simulations in general-relativistic hydrodynamics (HD) - where the jet is artificially powered via the injection of energy near the $\mathrm{BH}$ - and use these simulations to compare and contrast the properties of the MHD and HD jets.

\section{MHD VS HD JETS}

We employ BHAC to solve the general-relativistic MHD equations in a Kerr background spacetime (Porth et al. 2017). In order to describe the ejected matter and the torus around the compact remnant that was produced after a BNS merger, we follow the setup introduced in Nathanail et al. (2019) and additional information on the numerical setup are reported in the Appendix. The properties of the models simulated study are listed in Table 1.

HD jets have been thoroughly studied in the context of short GRBs from BNS mergers (Nagakura et al. 2014; Murguia-Berthier et al. 2014, 2016; Duffell et al. 2015, 2018). The MHD jets in our simulations are launched self-consistently over the timescale of the simulations, which ranges between $\sim 40 \mathrm{~ms}$ (for most cases) and $\sim 160 \mathrm{~ms}$. Overall, the dynamics of the plasma can be briefly described as follows: starting from a non self- gravitating torus with initial size $r_{\text {in }}=6 M=23.8 \mathrm{~km}$ and $r_{\text {out }}=14.3 M=56.7 \mathrm{~km}$ and containing a magnetic field of various topologies and strengths (cf., Table 1), the magnetorotational instability (MRI) develops, driving the accretion of matter and magnetic flux onto the BH. At the same time, the magnetic pressure in the torus expels the outer layers with an efficiency that depends strongly on the initial plasma $\beta$ parameter in the torus. As the MRI saturates and accretion reaches a steady state, the funnel region above the BH is cleared up and an MHD jet is formed. This accretion process can then continue until either the torus is accreted and ejected, or when the BH has lost much of its reducible energy by spinning down (Nathanail et al. 2016).

As the MHD jet breaks out from the ejecta that, in our setup, terminate at a radius of $1,200 \mathrm{~km}$, it enters in a region of low-density material where it does not encounter any matter pressure-gradient that contributed to its collimation. As a result, the jet expands in the transversal direction while maintaining a high degree of collimation. More precisely, when the head of the jet reaches $\sim 1,500 \mathrm{~km}$, the opening angles at a distance of $\sim 500 \mathrm{~km}$ and $\sim 1,500 \mathrm{~km}$ are $\theta_{\text {jet }} \simeq 13^{\circ}$ and $\theta_{\text {jet }} \simeq 15^{\circ}$, respectively. By the time the MHD jet reaches the outer boundary of the computational domain at $\sim 10,000 \mathrm{~km}$, the opening angle is still very small and $\theta_{\text {jet }} \simeq 13^{\circ}$. These values depend in detail on the initial conditions of the jet and on the properties of the ambient medium (Tchekhovskoy et al. 2008), but do not vary significantly in the simulations we have considered.

Another robust feature in all our MHD models reported in Table 1, is an almost hollow core subtending an angle $\theta_{\text {core }} \approx 4^{\circ}-5^{\circ}$, thus much smaller than the overall opening angle of the MHD jet, $\theta_{\text {jet }} \gtrsim 10^{\circ}$; the latter is consistent with numerical-relativity simulations where the starting point for the launching of such a jet is reached (Rezzolla et al. 2011; Kiuchi et al. 2014; Dionysopoulou 

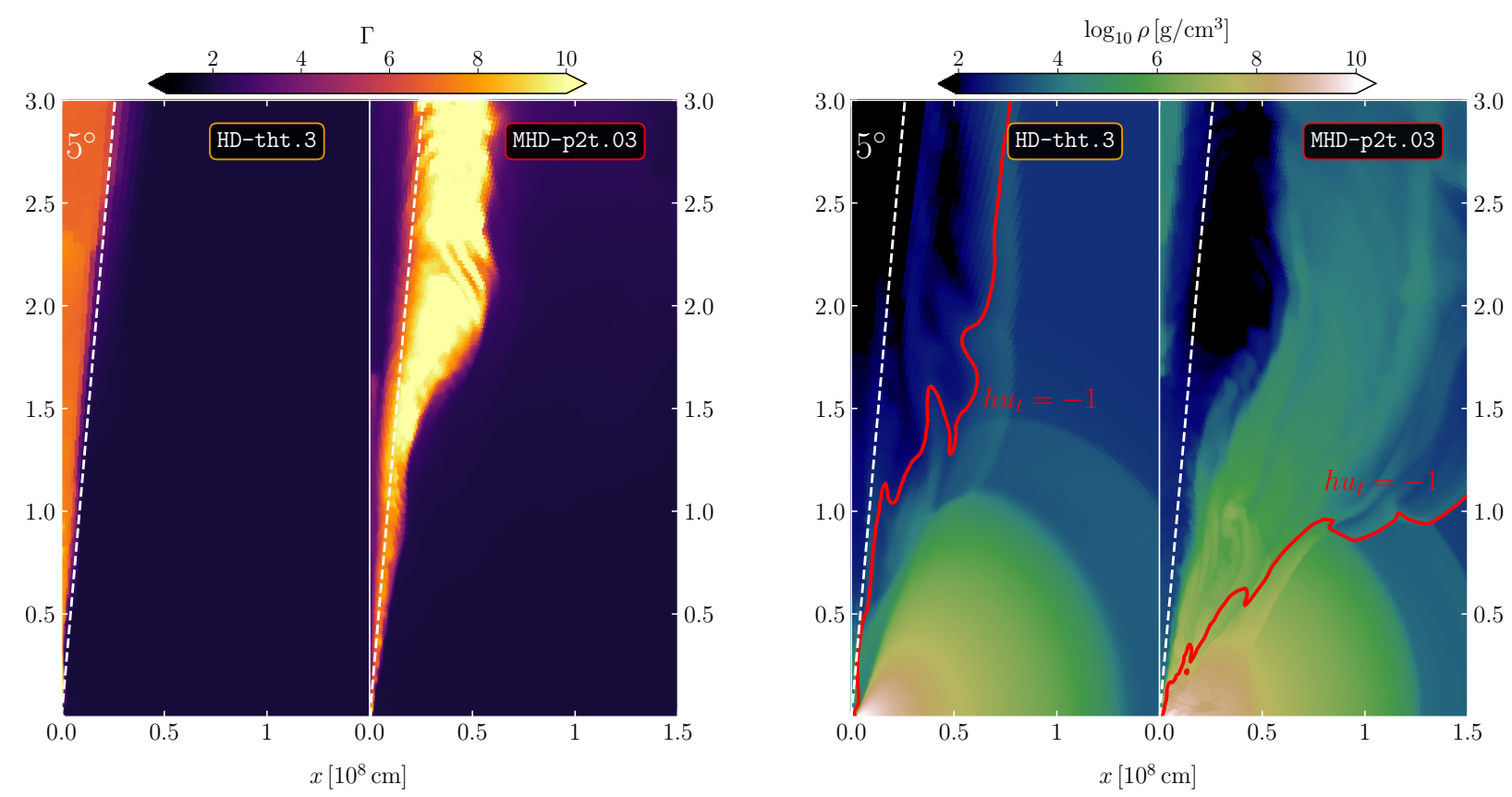

Figure 1. Lorentz factor (left panel) and density (right panel) distribution for two representative models: MHD-p2t.03 (left part of each plot) and HD-tht.3 (right part of each plot). The dashed white line indicates a cone with opening angle of $5^{\circ}$, highlighting the slow core of the MHD jet model. On the right panel the red lines denote the contour of $h u_{t}=-1$, so that matter above such line is gravitationally unbound; clearly the amount of ejected mass from the MHD jet is significantly larger than in the HD jet model.

et al. 2015; Kawamura et al. 2016; Ruiz et al. 2016)ํ․ In Fig. 1 we show a comparison between an MHD and a HD jet, where both jets have passed through the torus and the ejected matter. The Lorentz factor, shown on the left panel, clearly tends to unity in the inner core of the MHD jet. The appearance of a hollow cone in MHD jets has been pointed out previously in the literature (Komissarov et al. 2007; Tchekhovskoy et al. 2008; Lyubarsky 2009), but these were smaller than the one found here in our simulations inspired by BNS merger scenarios.

The structure and opening angle of the jet models presented in these studies depend strongly on the collimating agent. In the case of long GRBs, this agent is represented by the disk wind and the stellar layers that the jet has to bore. On the other hand, in the case of short GRBs produced from BNS mergers, once the jet breaks out from the matter ejected by the merger, it encounters the low-density interstellar medium (ISM), with number densities $n_{\text {ISM }} \sim 10^{-3}-10^{-1} \mathrm{~cm}^{-3}$, so that no significant further collimation is expected after breakout.

Duffell et al. (2018) have shown that as a HD jet drills through merger ejecta, it does not deposit significant energy, and thus has limited impact on the amount of ejected mass and the appearance of a kilonova. This is in stark contrast with what happens for MHD jets,

\footnotetext{
1 While "hollow core" is a standard denomination, the core of the jet does actually contain matter, but with very small Lorentz factor and energy.
}

the magnetized torus produces winds, with velocities far below the relativistic jet but significant enough that a large fraction of the initial matter distribution becomes unbound. On the right panel of Fig. 1 we show the distribution of the rest-mass density at time $t \sim 26 \mathrm{~ms}$, after the MHD and the HD jets have broken out from the merger ejecta. To quantify how much matter becomes unbound, we employ the Bernoulli criterion and assume a fluid element to be unbound if it has a Bernoulli constant $h u_{t} \leq-1$, where $h$ is the specific enthalpy of the fluid (Rezzolla \& Zanotti 2013). We then apply this criterion to measure the flux of unbound matter on a 2-sphere of $4,000 \mathrm{~km}$ and report in last two columns of Table 1 the amount of ejected mass and the fraction of the ejected mass with respect to the initial mass of the torus. Note that in all cases considered the ejected mass is between a few percent of the initial mass and up to a maximum of 37\%; furthermore, models with higher initial $\sigma$, have a larger fraction of unbound matter.

The angular structure of the HD and MHD jets can be better appreciated through the polar plots in Fig. 2, where we report the Lorentz factor and the energy, i.e., the volume integral up to the outer boundary of the total energy density, relative to the unbound material of three representative MHD jets and of the HD jet. The Lorentz factor (left panel of Fig. 2) is measured on a 2sphere with radius $r \sim 2,000 \mathrm{~km}$, and is integrated over a time interval of $\tau_{\text {avg }} \sim 4 \mathrm{~ms}$ to capture both the variability 

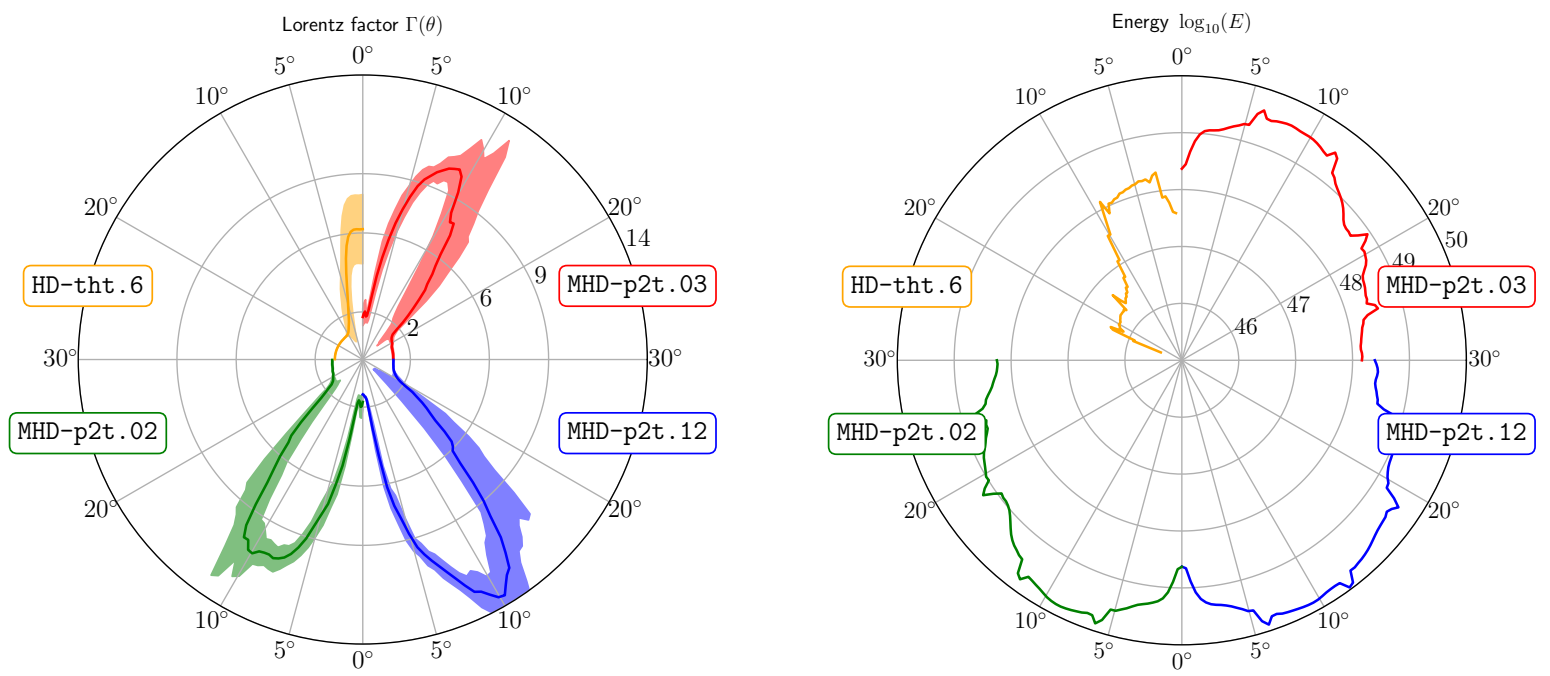

Figure 2. Upper panels: Polar plots of the Lorentz factor for four representative outflows over a quadrant (left panel (a)), or within a cone of $30^{\circ}$ (right panel (b)); the thick lines show the time-averaged values, while the shaded region the 1- $\sigma$ variance. Lower panel (c): Polar plot of the energy distribution for four representative models within a cone of $30^{\circ}$.
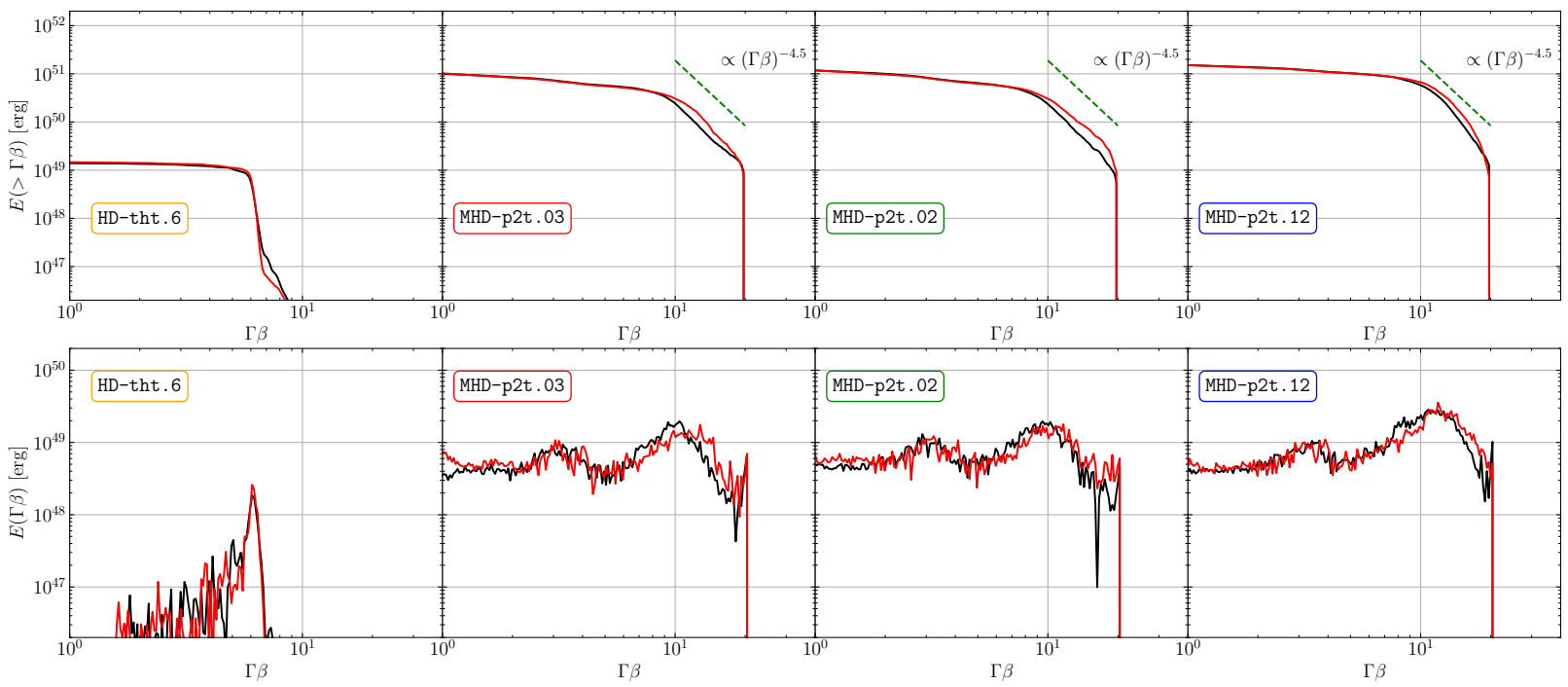

Figure 3. Energy distributions shown as either as $E=E(>\Gamma \beta)$ (top panels) or as $E=E(\Gamma \beta)$ (bottom panels) for the four representative models. The black and red solid lines represent the distribution at different times, $t=15$ and $20 \mathrm{~ms}$ respectively.

and the steady features.

Each of the four quadrants refers to one of the models considered, i.e., MHD-p2t.03, MHD-p2t.02, MHD-p2t.12, and HD-tht.6, with a thick line indicating the timeaveraged values and with the shaded areas showing the 1- $\sigma$ variance over the time interval $\tau_{\text {avg }}$, i.e., the $68 \%$ variation of the Lorentz factor at each angle. The right panel of Fig. 2, on the other hand, shows the angular distribution of the energy for the four models, where the energy is integrated for every angle for unbound matter with $\Gamma>1.2$; such a cut-off is introduced to avoid the inclusion of comparatively slow material.

In Fig. 3 we show instead the energy distribution above a certain value of $\Gamma \beta$, i.e., $E(>\Gamma \beta)$, as a function of $\Gamma \beta$, both for the HD jet and for the three representative MHD models. Since the energy $E$ generically grows with $\Gamma \beta$, the quantity $E(>\Gamma \beta)$ helps capture the nonlinear growth as a deviation from a constant value and to determine the cut-off at the highest energies. The energy is measured after the jet has broken out from the merger ejecta, i.e., $t=10 \mathrm{~ms}$, and is reported at three different times with a separation of $5 \mathrm{~ms}$ in time. Note that the HD jet 
is less powerful and with an energy that has an almost linear dependence $\Gamma \beta$ but to $\Gamma \beta \simeq 6-7$, when it has a very sharp fall-of profile at moderate Lorentz factors. Therefore, in a HD jet a most of the energy is concentrated in the fast-moving material.

On the other hand, all the MHD jets are up to two orders of magnitude more powerful and have a sub-linear growth of energy with $\Gamma \beta$; at the same time, the cut-off is less abrupt and preceded by a clear power-law fall-off at high Lorentz factors, which can be approximated as $E(>\Gamma \beta) \propto(\Gamma \beta)^{-4.5}$. Hence, in the case of MHD jets, most of the energy is at $\Gamma \beta \sim 10$, but the energy distribution in the plasma can reach very large values. Note that a cut-off of $\Gamma \simeq 20$ is set to avoid to account for portions of the flow where the accuracy of the numerical solution is reduced because of the large Lorentz factors reached.

It is worth noting that the bulk of our MHD jets is moving relatively fast and overall faster than what observed in other simulations (Gottlieb et al. 2018) or analytical modellings (Mooley et al. 2018a; Gill \& Granot 2018), where most of the energy is in slow-moving material and the power-law behaviour $\Gamma \beta^{-(4-5)}$ is seen already for $\Gamma \beta \simeq 1$. As a final remark, we note that since our MHD jets are launched as a result of GRMHD accretion processes, their energetics cannot be steered from the initial conditions, but is the self-consistent result of the simulations.

\section{AFTERGLOW EMISSION}

The afterglow emission is expected to be dominated by synchrotron radiation from electrons at the forward shock propagating into the low-density ISM and that are accelerated into a power-law energy distribution of the type $n_{e}\left(\Gamma_{e}\right) \propto \Gamma_{e}^{-p}$, where $n_{e}$ and $\Gamma_{e}$ are the number density and Lorentz factors of the electrons, respectively; hereafter, we will assume $p=2.16$, which is consistent with previous analysis for the afterglow of GRB170817A (Troja et al. 2019; Hajela et al. 2019). Following Sari et al. (1998), we model the emission that depends on the microphysical parameters $\epsilon_{e}$ and $\epsilon_{B}$, which describe the fraction of the total internal energy behind the shock given to electrons and to the magnetic field, respectively. The afterglow lightcurves are computed following the angular distributions of the Lorentz factor and of the energy profile (cf., Fig. 2), together with the energy distribution in $\Gamma \beta$ (cf., 3). The angular structure is binned uniformly in 200 angles along the $\theta$ direction, which yields the initial $\Gamma_{0}(\theta)$ and isotropic-equivalent energy $E_{\mathrm{iso}}(\theta)$ of the flow (see Granot et al. 1999; Gill \& Granot 2018, for details).

As representative examples of our fits, we make use of model HD-tht. 6 and model MHD-p2t.03. For the data, on the other hand, we employ the most recent afterglow data, i.e., $t \lesssim 743 \mathrm{~d}$ after merger (see, e.g., Hajela et al.

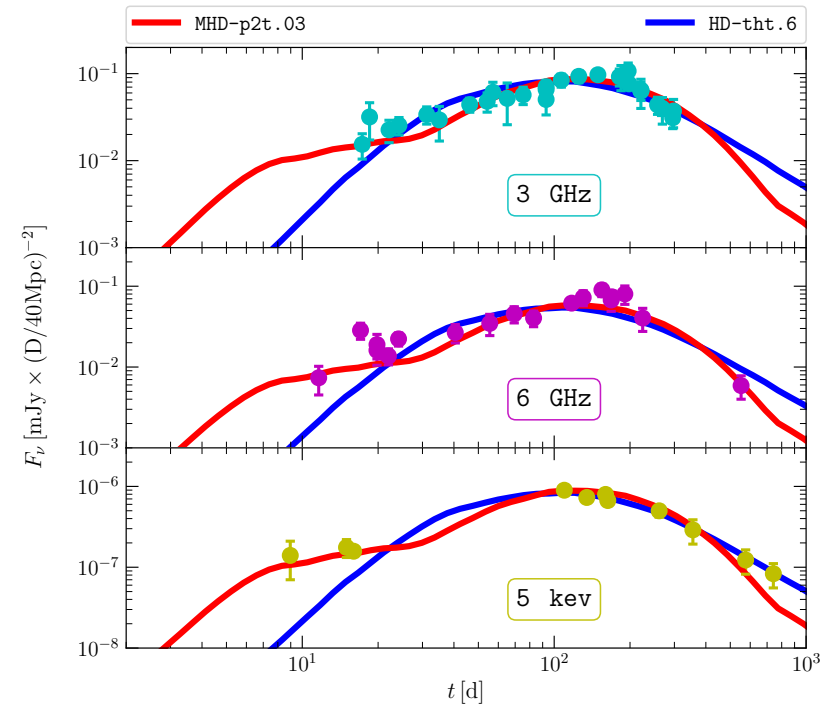

Figure 4. Broad band observations of GRB170817A with the best-fit lightcurves of models MHD-p2t.03 (red line; see main text for the fitting parameters) and HD-tht. 6 (dashed blue line; see main text for the fitting parameters).

2019, for the latest observations in X-rays) consisting of $\mathrm{X}$-ray emission at $5 \mathrm{keV}$ and VLA radio observations at 3 and $6 \mathrm{GHz}$ (Margutti et al. 2017, 2018; Alexander et al. 2017, 2018; Hallinan et al. 2017; Mooley et al. 2018a,c; Dobie et al. 2018; Troja et al. 2018, 2019; Hajela et al. 2019). The fit is performed with five free parameters, namely: the observer angle $\theta_{\mathrm{obs}}$, the energy of the burst $E$, the fraction of the total energy in the electrons $\epsilon_{e}$, the fraction of the total energy in the magnetic field $\epsilon_{B}$, and the circum-merger density, $n_{\text {ISM }}$. Note that the parameter space is degenerate since the model parameters outnumber the available constraints from the data (see, e.g., Gill et al. 2019). The best-fit parameters are then found using a genetic algorithm to optimize the parameter selection and minimize the reduced $\chi_{v}^{2}$ (Fromm et al. 2019), while the fitting procedure is applied simultaneously to the three different bands.

The afterglow lightcurves relative to the set of parameters providing the best fits for the two models MHDp2t.03 and HD-tht.6, along with the observational data, are shown in Fig. 4 for a source at $40 \mathrm{Mpc}$, where the upper and middle panels correspond to radio observations at 3 and $6 \mathrm{GHz}$, while the lower panel to X-ray observations at $5 \mathrm{keV}$.

Overall, the MHD jet model MHD-p2t.03 yields a better fit to the data, with a reduced $\chi_{v}^{2}=2.5$ and parameters $\theta_{\mathrm{obs}}=21.5^{\circ}, E=10^{50.85} \mathrm{erg}, \log _{10}\left(\epsilon_{e}\right)=$ $-0.99, \log _{10}\left(\epsilon_{B}\right)=-4.4$, and $n_{\mathrm{ISM}}=10^{-2.04} \mathrm{~cm}^{3}$ (red line). It captures well the first data points in the afterglow, together with the peak and the fall-off. The HD jet model HD-tht.6, on the other hand, provides a less-good fit 
with reduced chi-squared are $\chi_{v}^{2}=4.04$ and parameters $\theta_{\text {obs }}=21.4^{\circ}, E=10^{51.01} \mathrm{erg}, \log _{10}\left(\epsilon_{e}\right)=-0.27, \log _{10}\left(\epsilon_{B}\right)=$ -2.8 , and $n_{\mathrm{ISM}}=10^{-4.14} \mathrm{~cm}^{3}$ (dashed blue line); however, it also yields a better match to the very late decay in the X-ray emission till 743 days after the merger (model HD-tht. 3 has $\chi_{v}^{2}=5.06$ and an HD jet with $\theta_{\text {jet }}=16^{\circ}$ has even larger reduced chi-squared). Interestingly, both of the best-fit models suggest an observation angle $\theta_{\text {obs }} \simeq 21^{\circ}$, which can then be taken as a robust feature of the emission of GRB170817A. Our estimates are thus consistent with those of Mooley et al. (2018b); Troja et al. (2019), and smaller than those coming from the semi-analytical and analytical modelings, which suggest instead $\theta_{\mathrm{obs}} \simeq 30^{\circ}$ (Hajela et al. 2019).

It is worth noting that when all the physical parameters - i.e., $E, \epsilon_{e}, \epsilon_{B}$, and $n_{\mathrm{ISM}}$ - are kept the same, the HD/MHD light curves show a marked difference. Indeed, while both lightcurves have similar power-law rise and fall-offs, the evolution of peak-times are considerably different, with the HD having a monotonic dependence of the peak-times with the viewing angle, with peak-times increasing as viewing angles become larger. The MHD lightcurves, instead, do not have a minimum peak-time at the smallest viewing angle, but for $\theta_{\mathrm{obs}} \gtrsim \theta_{\text {core }}$; the peaktime then increases steeply as the viewing angle grows. This considerable difference between the two afterglow lightcurves disappears for larger angles, that is, when the jets are observed off-axis.

\section{CONCLUSIONS}

We have performed a number of general-relativistic HD and MHD simulations to model the launching of a jet after a BNS merger and contrast the dynamics and appearance of HD and MHD jets. Overall, we find that:

(i) MHD jets have an intrinsic energy and velocity structure in the polar direction characterised by a "hollow core" subtending an angle $\theta_{\text {core }} \approx 4^{\circ}-5^{\circ}$ and an opening angle of $\theta_{\text {jet }>} \gtrsim 10^{\circ}$. HD jets, on the other hand, have a uniform energy and polar structure and much smaller opening angles of $\theta_{\text {jet }} \sim 3^{\circ}$. (ii) MHD jets eject significant amounts of matter, amounting to $\lesssim 30 \%$ of the total mass of the system and about two orders of magnitude more than HD jets. (iii) The energy stratification in MHD jets naturally yields the power-law energy scaling $E(>\Gamma \beta) \propto(\Gamma \beta)^{-4.5}$ often introduced in analytical modelling. This feature is robust and does not require special tuning as is the case instead for HD jets. (iv) MHD jets provide fits to the afterglow data from GRB170817A in three different bands $(3 \mathrm{GHz}, 6 \mathrm{GHz}$ and $5 \mathrm{keV})$ that are not only very good but also comparatively better than those of the HD jets. While even better fits can be constructed with suitably constructed HD jets, the fit obtained with MHD jets is robust and without free parameters. (v) Both of the best-fit HD/MHD models suggest an observation angle $\theta_{\mathrm{obs}} \simeq 21^{\circ}$ for GRB170817A.

While this is arguably the most comprehensive exploration of jet launching from BNS mergers, explore and contrasting for the first time HD and MHD jets, future work will have to include additional jet models, a closer comparison with other models proposed in the literature, and a step towards imaging in the radio band.

Acknowledgements. Support comes in part also from "PHAROS", COST Action CA16214 and the LOEWEProgram in HIC for FAIR. The simulations were performed on the SuperMUC cluster at the LRZ in Garching, on the LOEWE cluster at the CSC in Frankfurt, and on the HazelHen cluster at the HLRS in Stuttgart.

\section{REFERENCES}

Abramowicz M., Jaroszynski M., Sikora M., 1978, Astron. Astrophys., 63, 221

Alexander K. D., et al., 2017, Astrophys. J. Letters, 848, L21 Alexander K. D., et al., 2018, Astrophys. J. Letters, 863, L18 Bovard L., Martin D., Guercilena F., Arcones A., Rezzolla L., Korobkin O., 2017, Phys. Rev. D, 96, 124005

Bromberg O., Tchekhovskoy A., Gottlieb O., Nakar E., Piran T., 2018, Mon. Not. R. Astron. Soc., 475, 2971

D'Avanzo P., et al., 2018, A\&A, 613, L1

Dietrich T., Ujevic M., Tichy W., Bernuzzi S., Brügmann B., 2017, Phys. Rev. D, 95, 024029

Dionysopoulou K., Alic D., Rezzolla L., 2015, Phys. Rev. D, 92 , 084064

Dobie D., et al., 2018, Astrophys. J. Letters, 858, L15

Duffell P. C., Quataert E., MacFadyen A. I., 2015, Astrophys. J., 813,64

Duffell P. C., Quataert E., Kasen D., Klion H., 2018, preprint, (arXiv:1806.10616)

Fernández R., Tchekhovskoy A., Quataert E., Foucart F., Kasen D., 2018, Mon. Not. R. Astron. Soc.,

Fishbone L. G., Moncrief V., 1976, Astrophys. J., 207, 962

Foucart F., O'Connor E., Roberts L., Kidder L. E., Pfeiffer H. P., Scheel M. A., 2016, Phys. Rev. D, 94, 123016

Fromm C. M., et al., 2019, Astron. Astrophys., 629, A4

Fujibayashi S., Kiuchi K., Nishimura N., Sekiguchi Y., Shibata

M., 2018, Astrophys. J., 860, 64

Geng J.-J., Zhang B., Kölligan A., Kuiper R., Huang Y.-F., 2019, Astrophys. J. Letters, 877, L40

Ghirlanda G., et al., 2019, Science, 363, 968

Gill R., Granot J., 2018, Mon. Not. R. Astron. Soc.,

Gill R., Granot J., De Colle F., Urrutia G., 2019, arXiv:1902.10303, e-prints,

Goldstein A., et al., 2017, Astrophys. J. Letters, 848, L14

Gottlieb O., Nakar E., Piran T., 2018, Mon. Not. R. Astron. Soc., 473,576

Granot J., Piran T., Sari R., 1999, Astrophys. J., 513, 679

Hajela A., et al., 2019, Astrophys. J. Lett., 886, L17

Hallinan G., et al., 2017, Science, 358, 1579

Kasliwal M. M., et al., 2017, Science, 358, 1559

Kathirgamaraju A., Barniol Duran R., Giannios D., 2018, Mon. Not. R. Astron. Soc., 473, L121

Kathirgamaraju A., Tchekhovskoy A., Giannios D., Barniol

Duran R., 2019, Mon. Not. R. Astron. Soc., 484, L98

Kawamura T., Giacomazzo B., Kastaun W., Ciolfi R., Endrizzi

A., Baiotti L., Perna R., 2016, Phys. Rev. D, 94, 064012 
Kiuchi K., Kyutoku K., Sekiguchi Y., Shibata M., Wada T., 2014 Phys. Rev. D, 90, 041502

Kiuchi K., Kyutoku K., Sekiguchi Y., Shibata M., 2018, Phys. Rev. D, 97, 124039

Komissarov S. S., Barkov M. V., Vlahakis N., Königl A., 2007, Mon. Not. R. Astron. Soc., 380, 51

Lazzati D., Perna R., Morsony B. J., Lopez-Camara D., Cantiello M., Ciolfi R., Giacomazzo B., Workman J. C., 2018, Phys. Rev. Lett., 120, 241103

Lyman J. D., et al., 2018, Nature Astronomy,

Lyubarsky Y., 2009, Astrophys. J., 698, 1570

Margutti R., et al., 2017, Astrophys. J. Letters, 848, L20

Margutti R., et al., 2018, Astrophys. J. Letters, 856, L18

Mooley K. P., et al., 2018a, Nature, 554, 207

Mooley K. P., et al., 2018b, Nature, 561, 355

Mooley K. P., et al., 2018c, Astrophys. J. Lett., 868, L11

Murguia-Berthier A., Montes G., Ramirez-Ruiz E., De Colle F., Lee W. H., 2014, Astrophys. J., 788, L8

Murguia-Berthier A., et al., 2016, Astrophys. J. Lett., 835, L34

Nagakura H., Hotokezaka K., Sekiguchi Y., Shibata M., Ioka K., 2014, Astrophys. J., 784, L28

Nathanail A., Strantzalis A., Contopoulos I., 2016, Mon. Not. R. Astron. Soc., 455, 4479

Nathanail A., Porth O., Rezzolla L., 2019, Astrophys. J. Lett, 870, L20

Porth O., Olivares H., Mizuno Y., Younsi Z., Rezzolla L., Moscibrodzka M., Falcke H., Kramer M., 2017, Computational Astrophysics and Cosmology, 4, 1
Radice D., Galeazzi F., Lippuner J., Roberts L. F., Ott C. D., Rezzolla L., 2016, Mon. Not. R. Astron. Soc., 460, 3255

Rezzolla L., Zanotti O., 2013, Relativistic Hydrodynamics. Oxford University Press, Oxford, UK,

doi:10.1093/acprof:oso/9780198528906.001.0001

Rezzolla L., Giacomazzo B., Baiotti L., Granot J., Kouveliotou

C., Aloy M. A., 2011, Astrophys. J. Letters, 732, L6

Ruiz M., Lang R. N., Paschalidis V., Shapiro S. L., 2016, Astrophys. J. Lett., 824, L6

Sari R., Piran T., Narayan R., 1998, Astrophys. J. Lett., 497, L17

Savchenko V., et al., 2017, Astrophys. J. Letters, 848, L15

Sekiguchi Y., Kiuchi K., Kyutoku K., Shibata M., Taniguchi K., 2016, Phys. Rev. D, 93, 124046

Tchekhovskoy A., McKinney J. C., Narayan R., 2008, Mon. Not. R. Astron. Soc., 388, 551

The LIGO Scientific Collaboration The Virgo Collaboration 2017, Phys. Rev. Lett., 119, 161101

The LIGO Scientific Collaboration et al., 2017, Astrophys. J. Lett., 848, L12

Troja E., et al., 2017, Nature, 551, 71

Troja E., et al., 2018, Mon. Not. R. Astron. Soc., 478, L18

Troja E., et al., 2019, Mon. Not. R. Astron. Soc., 489, 1919

\section{APPENDIX}

\section{A. NUMERICAL SETUP AND MHD MODELS}

In this Appendix we provide details of the numerical setup of our simulations and further show results for an extensive selection of MHD models in order to check the robustness of the results. As anticipated, we use BHAC to solve the general-relativistic MHD equations in a Kerr background spacetime (Porth et al. 2017). To mimic the post-merger remnant in GW170817 and as initial condition for the launching of an MHD jet, we consider a non self-gravitating torus (Fishbone \& Moncrief 1976; Abramowicz et al. 1978) around a BH of mass $M=2.7 M_{\odot}$ and various dimensionless spins (see Table 1). The radial extent of the initial matter distribution is set to be $1,200 \mathrm{~km}$, in order to account for the expansion of the torus, and also for the matter expelled during merger, which has reached such a distance. To accommodate such a large extension of matter, the numerical domain has always a radius of $10,000 \mathrm{~km}$. Since we here focus on the production and launch of a jet, at the beginning of the simulation all matter is bound and set to have a zero velocity. However, we do measure the mass that becomes unbound as a result of the jet launching and compute its contribution to the kilonova at the end of the simulation. The simulations are performed in two spatial dimensions using a spherical polar coordinate system. The computational domain is resolved with either $1024 \times 512$ or $512 \times 256$ cells and with three refinement levels, thus yielding an effective resolution of $4092 \times 2048$ cells.

Over the past several years, a robust picture has been drawn on the distribution of the ejected matter after the merger. More specifically, BNS merger simulations indicate that the polar region is not entirely empty of matter (Sekiguchi et al. 2016; Foucart et al. 2016; Radice et al. 2016; Bovard et al. 2017; Dietrich et al. 2017; Fujibayashi et al. 2018). To reproduce such conditions, we fill the polar region with matter, having density that is 2.5 orders of magnitude less than the maximum density of the torus and a radial profile that scales like $r^{-1.5}$, with an exception for model HD-tht.6, where the matter in the polar region has 1 order of magnitude higher density, but has the same radial profile. In a typical BNS merger, the two stars have a mildly strong initial magnetic field, which is expected to be amplified during merger, either via the Kelvin-Helmholtz or the magnetorotational instability, yielding a very magnetic energy $>10^{50} \mathrm{erg}$, and with ratio between poloidal and the toroidal components that is $\approx 0.3$ (Kiuchi et al. 2018). To reproduce the enhancement in the magnetic field after the merger, we initialize our simulations with a poloidal nested-loop magnetic field structure and a toroidal component that traces the fluid pressure; by tuning the strength of two components it is then possible to obtain the desired ratio in the corresponding magnetic energies.

To explore a space of parameters that is as wide as reasonably possible, we vary the initial magnetic field, the ratio of the poloidal-to-toroidal magnetic-field energy, the spin of the BH, as well as the size and morphology of the torus 


\begin{tabular}{|c|c|c|c|c|c|c|c|c|c|c|c|c|c|c|}
\hline model & $\begin{array}{c}L \\
{[\mathrm{erg} / \mathrm{s}]}\end{array}$ & $\begin{array}{l}t_{\mathrm{inj}} \\
{[\mathrm{s}]}\end{array}$ & $\Gamma_{\text {init }}$ & $\begin{array}{c}\theta_{\text {jet }} \\
{[\mathrm{deg}]}\end{array}$ & $\begin{array}{l}E_{B_{\phi}} \\
{[\text { erg] }} \\
10^{49}\end{array}$ & $\begin{array}{c}E_{B_{\mathrm{p}}} \\
{[\mathrm{erg}]} \\
10^{49}\end{array}$ & $\frac{E_{B_{p}}}{E_{B_{\phi}}}$ & $\sigma_{\max }$ & $\beta_{\min }$ & $\begin{array}{c}\rho_{\max } \\
{\left[\mathrm{g} / \mathrm{cm}^{3}\right]} \\
10^{10}\end{array}$ & $a$ & $\begin{array}{c}M_{\mathrm{tot}} \\
{\left[M_{\odot}\right]}\end{array}$ & $\begin{array}{c}M_{\mathrm{ej}} \\
{\left[M_{\odot}\right]}\end{array}$ & $\begin{array}{c}\frac{M_{\mathrm{ej}}}{M_{\mathrm{tot}}} \\
\%\end{array}$ \\
\hline HD-tht. 6 & $10^{51}$ & 0.1 & 10 & 6 & - & - & - & - & - & 1.5 & 0.9375 & 0.108 & 0.0001 & 0.12 \\
\hline HD-tht. 3 & $10^{51}$ & 0.1 & 10 & 3 & - & - & - & - & - & 1.5 & 0.9375 & 0.108 & 0.0001 & 0.13 \\
\hline MHD-p2t.03 & - & - & - & - & 5.0 & 1.6 & 0.3 & 0.065 & 0.13 & 1.5 & 0.9375 & 0.108 & 0.039 & 36.0 \\
\hline MHD-p2t.03-LB & - & - & - & - & 0.36 & 0.28 & 0.3 & 0.0026 & 3.20 & 2.0 & 0.9375 & 0.144 & 0.021 & 1.45 \\
\hline MHD-p2t. 02 & - & - & - & - & 10 & 2.1 & 0.2 & 0.065 & 0.13 & 2.0 & 0.9375 & 0.144 & 0.053 & 37.1 \\
\hline MHD-p2t.02-LB & - & - & - & - & 0.4 & 0.084 & 0.2 & 0.002 & 3.25 & 2.0 & 0.9375 & 0.144 & 0.002 & 1.60 \\
\hline MHD-p2t. 12 & - & - & - & - & 1.2 & 1.5 & 1.2 & 0.036 & 0.13 & 1.5 & 0.9375 & 0.108 & 0.036 & 34.1 \\
\hline MHD-p2t. 04 & - & - & - & - & 4.1 & 1.6 & 0.4 & 0.065 & 0.13 & 1.5 & 0.9375 & 0.108 & 0.033 & 31.2 \\
\hline MHD-a.8-LB & - & - & - & - & 0.19 & 0.115 & 0.6 & 0.0024 & 3.30 & 3.0 & 0.8 & 0.118 & 0.0034 & 2.10 \\
\hline MHD-a. 8-MB & - & - & - & - & 1.7 & 1.05 & 0.6 & 0.02 & 0.36 & 3.0 & 0.8 & 0.118 & 0.014 & 12.0 \\
\hline MHD-a. 8 & - & - & - & - & 3.9 & 2.4 & 0.6 & 0.06 & 0.13 & 2.5 & 0.8 & 0.098 & 0.029 & 29.8 \\
\hline MHD-rout-52.4 & - & - & - & - & 1.0 & 0.195 & 0.2 & 0.0016 & 4.10 & 10 & 0.9375 & 0.121 & 0.018 & 15.6 \\
\hline MHD-600km & - & - & - & - & 1.7 & 0.54 & 0.3 & 0.016 & 0.52 & 2.0 & 0.9375 & 0.127 & 0.0077 & 6.23 \\
\hline MHD-900km & - & - & - & - & 1.3 & 0.4 & 0.3 & 0.016 & 0.52 & 1.5 & 0.9375 & 0.106 & 0.004 & 3.94 \\
\hline
\end{tabular}

Table A2:. Properties of the various HD and MHD jets considered: luminosity of the HD jet $(L)$, duration of the HD injection $\left(t_{\text {inj }}\right)$, initial Lorentz factor of the HD jet $\left(\Gamma_{\text {init }}\right)$, initial opening angle of the HD jet $\left(\theta_{\text {jet }}\right)$, toroidal and poloidal magnetic energies $\left(E_{B_{\phi}}, E_{B_{\mathrm{p}}}\right)$ and their ratio, maximum magnetization in the torus $\left(\sigma:=B^{2} / 4 \pi \rho\right)$, minimum plasma parameter in torus $\left(\beta:=p / p_{m}\right.$, where $p$ and $p_{m}$ are the fluid and magnetic pressures respectively), maximum density of the torus $\left(\rho_{\max }\right)$ and dimensionless spin parameter of the BH $\left(a:=J / M^{2}\right)$, initial total mass $\left(M_{\mathrm{tot}}\right)$, ejected mass $\left(M_{\mathrm{ej}}\right)$ and their ratio. For all models the initial torus parameters are $r_{\mathrm{in}}=23.8 \mathrm{~km}, r_{\text {out }}=56.8 \mathrm{~km}$ and the matter distribution has a radial extent till $r_{\text {ext }}=1,200 \mathrm{~km}$, whereas model MHD-rout-52.4 has $r_{\text {out }}=52.4 \mathrm{~km}$, model MHD-600km has $r_{\mathrm{ext}}=600 \mathrm{~km}$ and MHD-900km has $r_{\mathrm{ext}}=900 \mathrm{~km}$. Note that models ending with MB and LB refer to matter with a medium and low magnetic-field strength, respectively, while all the other quantities are held the same.

(which is ultimately dictated by the spin of the BH). The details of all the models used are listed in Table 2. For illustrative purposes, we report in Fig. 5 the angular structure of eight outflows from Table 2, showing the Lorentz factor within an angle of $0 \leq \theta \leq 30^{\circ}$. Similar to Fig. 2, the Lorentz factor (thick line) is measured in slices of constant radius, i.e., $r \sim 2000 \mathrm{~km}$, and integrated over a time interval of $\tau_{\text {avg }} \sim 2 \mathrm{~ms}$, the shaded areas show the 1- $\sigma$ variance over the time interval $\tau_{\text {avg }}$, i.e., the $68 \%$ variation of the Lorentz factor at each angle. From the two polar plots it is evident that the presence of a hollow core with an opening of $\approx 4^{\circ}-5^{\circ}$ is robust in all of the MHD models considered in our study. 

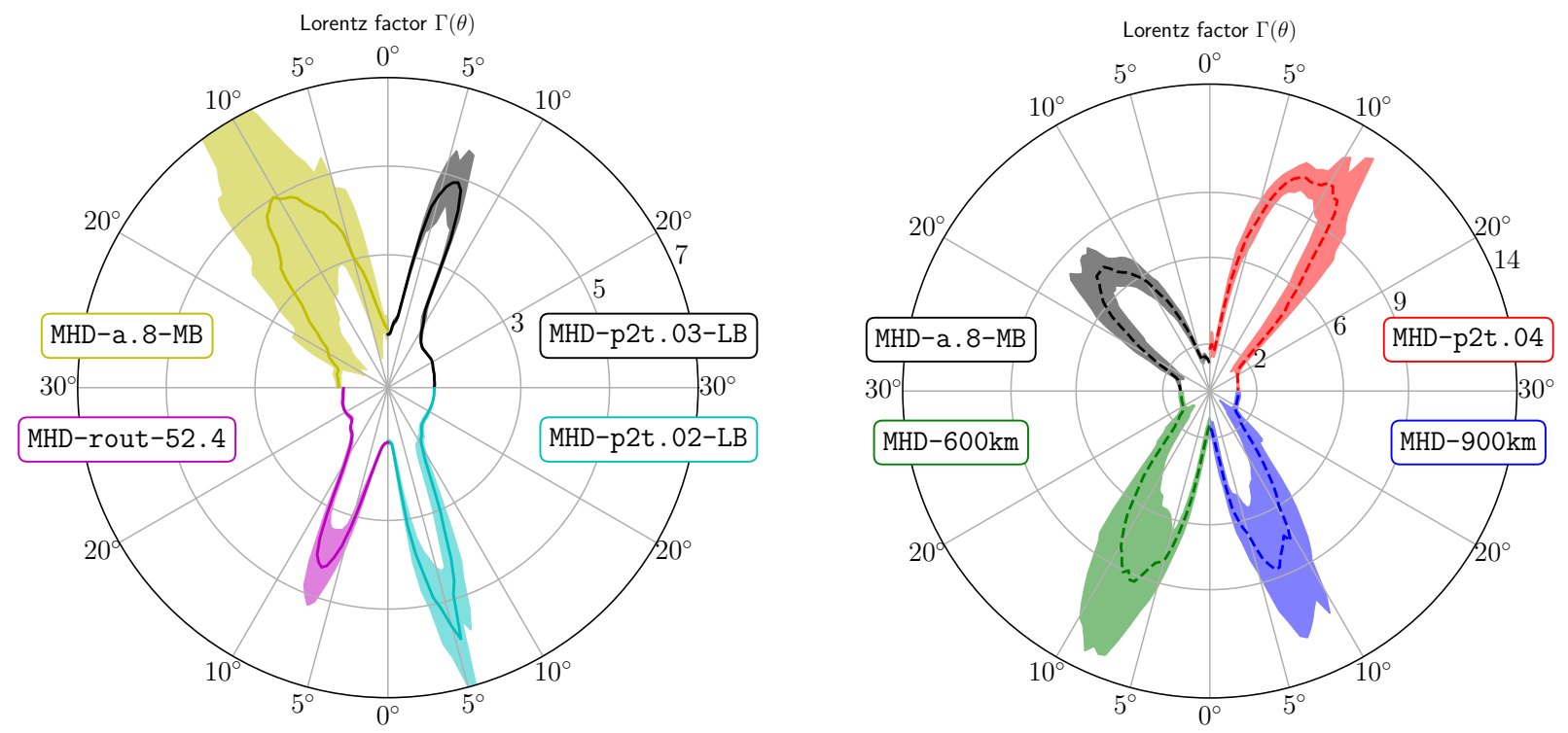

Figure A5:. Polar plots of the Lorentz factor for eight outflows from Table 2 within a cone of $30^{\circ}$, the thick lines show the average values, while the shaded region the $1-\sigma$ variance. 